\title{
Is Alzheimer's Disease an Irreversible Loss of Memory Function with No Hope of Return?
}

\author{
Jean-Louis Kraus \\ Biomolecular Chemistry Team, IBDM (Institut de Biologie du Développement de Marseille Luminy), UMR 7288 \\ CNRS-AMU (Aix Marseille University), Parc Scientifique de Luminy BP 907, Marseille, France \\ Email: jean-louis.kraus@univ-amu.fr
}

Received 18 December 2013; revised 16 January 2014; accepted 15 February 2014

Copyright (C) 2014 by author and Scientific Research Publishing Inc.

This work is licensed under the Creative Commons Attribution International License (CC BY).

http://creativecommons.org/licenses/by/4.0/

\section{c) (i) Open Access}

\begin{abstract}
Since Socrates memory has always been an intriguing matter. To date for the common run of people being hit by Alzheimer's disease (AD) is "memory loss with no hope of return". Looking at the brain matter as an ordered closed system where memory and cognition information are located, according to Loschmidt Paradox, and thermodynamically speaking, it should be possible for AD patient brains presenting severe cognitive impairments (disordered state), to move backward to the original brain ordered state. This assumption based on thermodynamic concepts may appear inconsistent with current knowledge in neurosciences on "memory and its operation". Attempts to connect neurobiological science to quantum physics concepts may allow a breakthrough in the understanding of memory function and therefore a step ahead in the knowledge of how memory works.
\end{abstract}

\section{Keywords}

Insert Memory, Loschmidt Paradox, Matter Constitution

\section{Introduction}

Imagine a "pure $A D$ patient” only suffering of advanced Alzheimer's Disease, for whom no other pathologies related to any other main organ dysfunction have been diagnosed. Only his learning and memory functions are affected, resulting in severe cognitive impairments. For this "pure AD patient", any therapeutic treatment aiming to cure his disease should target in priority the specific parts of his brain where are located both the storage and the release of memory. Central Nervous System does not have identified places for memory "storage and/or release”, the system seems to work in a complex set of interrelated and highly unpredictable networks. Nevertheless, recent studies seem to agree that memories are made presumably in the constitutive matter of two specific 
brain regions: cerebral cortex (Hasan et al., 2013) or/and “hippocampus” (Pavlopoulos et al., 2013).

\section{Memory and Loschmidt Paradox}

Considering the matter of these brain regions as "ordered closed systems" where memory is localized, it could be postulated that in AD patients the system represented by cerebral cortex and/or hippocampus regions is highly disordered. According to the well known "Loschmidt paradox" (Loschmidt, 1876; Cucic, 2009), which stated that entropy (disorder) of any closed system which has evolved from an ordered state to a disordered state, should be able to return to its initial ordered state, simply in reversing the motion of the constitutive system particles without kinetic change of energy during the time of the inversion process. In other words, from a fundamental point of view, physicists consider that a closed system of ordered particles, which have evolved towards an highly disordered state, can be brought backward to the ordered state.

A significant example illustrating this paradox can be found in mineral salts crystallization process. Sodium chloride crystallizes as an orderly cubic form, in which each ion is surrounded by six ions of the opposite charge; after dissolution of the crystals in water and evaporation (Sirdeshmukh et al., 2001), according to Loschmidt paradox the new formed crystals kept their initial ordered cubic form; indicating that at the atomic level, the notion of memory is already contained.

Referring to Loschmidt's paradox, the closed system represented by the constitutive matter of the cerebral cortex or/and hippocampus should kept in "memory" its initial ordered state. Therefore thermodynamically speaking, it should be possible for $\mathrm{AD}$ patient disordered brains to move backward to the original brain ordered state.

\section{Where Are Stored Our Souvenirs?}

Socrates explained in the Thaetetus dialogue (Bostock, 1988) that mind could be seen as a wax box on which we stamp what we perceive or conceive. Whatever is impressed upon the wax we remember and know, so long as the image remains in the wax; whatever is obliterated or cannot be impressed, we forget and do not know. "When the wax we have in our soul is deep, abundant, smooth and well kneaded, the prints are deep enough, last longer, and men who have such souls, ease of learning, have a good memory and form true judgments".

There is no issue here to assimilate cerebral cortex and hippocampus regions, as Plato's wax box; however, the metaphor of the wax box used by Plato, already highlights the metaphysical question of the importance of the nature of the support on which memory is printed. Through my reasoning, I can already hear rumbling voices of biologists that oppose this pure thermodynamic concept of the brain memory arguing that molecular biology for several decades has highlighted the central role of neuronal death in neurodegenerative diseases, arguing that taken into account the post-mitotic nature of neurons, loss of neurons represents an irreversible process that inevitably leads to the irreversible loss of memory function, without the possibility of return to the initial structure. At this point can I humbly express the following hypothesis?

Could it be possible that information contained in memory of AD brain patients are not erased, they still exist, but the network of connections which has been partially destroyed by the loss of neurons, is no longer operational to allow transduction of the information printed in the regions of cerebral cortex and hippocampus?

\section{Constitution and Organization of the Constitutive Matter of Brain and Memory}

At this point a crucial question rises "In what the atomic constitution and organization of the matter of the cerebral cortex or hippocampus area are distinct from that of the other parts of the brain which are not related to memory and information storage? Answer to this question is desperately risky. Modern theories of physics of matter based on quantum mechanics concepts can be evoked.

- Presence of ferromagnetic material in a variety of tissues from the human brain which could function as magnetic tape bands (Kirschvink et al., 1992).

- Perception, reasoning, intuition and knowledge (cognition) are connected and are blended within the field as quantum memory (Kak, 2013).

- Memory could be encoded in biophotons having zero mass, no electric charge and an indefinitely long lifetime (Cifra et al., 2010).

However, the boundaries between what constitutes matter and everything else remains as vague as the de- 
marcation problem of delimiting science from everything else. Without indulging in a classic reductionist fallacy, answer this question still needs to understand how the intimate molecular structure (atoms and their nucleus intimate particles) of the human brain records information and how this matter acquires memory function capacity and cognitive functions. Let's consider the simple biological reactions which occur when the human brain is subjected to various types of psychological stress such as tears which appear shortly after learning the death of a parent. Tears are the result of a process which first requires the defunct remembrance by the memory of the weeper, followed by a well known biological process leading to tear production through lacrimal glands. But how the external stress stimulus expressed through the sound waves of the words used to announce the death, affects the molecular organization of the brain of the person touched by this mourning, is not clearly understood.

At this point the sense of memory should be recalled: Etymologically speaking "memory", taken in its latin sense of "memoria", has four different significances. The first corresponds to the idea of "having a very good memory; the second "to keep in memory" includes the notion of souvenir, the third sense is based on the "period of time covered or evoked by the souvenir", and the last significance seen as "monuments dedicated to the memories of human beings "is directly connected to the body of the man. Taken into account the significances of memory one can ask: "What are the relationships between the spirit and the memory?" An attempt to answer this question could be brought by Bergson (Bergson, 1896), who considers memory as the privileged point of the articulation between the spirit and the matter. "The spirit borrows, from the matter, perceptions, from where it takes its food, and thanks to memory where spirit has printed its freedom, those perceptions are translated into movements".

\section{To Cure Deficit Memory "Thermodynamic Consequences"}

Supposing a way to reverse the disordered state of the cerebral cortex matter towards its initial ordered state, allowing pure $A D$ patients to recover their full memory faculties including the four significances of the word memoria. This discovery not only has significant benefits in the treatment of one of the most devastating disease of the XXI century but also opens a possibility to impair or to interfere within the human being aging process. Modified human aging process would be the first attempt to deregulate human internal genetic clock with the hope of increasing the life of human being. Let's recall that from immemorial time, man has always dreamed of being immortal.

From a thermodynamicist point of view, probably not shared by neurobiologists, healing of patients' cognitive diseases $(A D)$, whatever the therapies used, will inevitably affect the entropy of the system represented by the intimate structure of the matter where memory and cognition are printed and stored: A recent argument seems to support this thermodynamic memory concept. According to Bérut et al. (Bérut et al., 2012) there is an intimate link between information theory and thermodynamics, since a tiny amount of heat is released when an individual bit of data is erased. Therefore any medical advances aiming to cure deficit memory, could be seen as "anti-aging escape velocity”, which according to De Grey (De Grey \& Rae, 2007) will postpone aging and will extend lifespan faster than the passage of time.

\section{Acknowledgements}

URMA association (Unis pour la Recherche sur la Maladie d'Alzheimer, Mrs M Pavone) and IBDML and CNRS are greatly acknowledged for financial support.

\section{References}

Bergson, H. (1896). Essay on the Relation of Body and Spirit. (Translators N. M. Paul and W. S. Palmer). Matière et Mémoire. Zone Books.

Bérut, A., Arakelyan, A., Petrosyan, A., Ciliberto, S., Dillenschneider, R., \& Lutz, E. (2012). Experimental Verification of Landauer's Principle Linking Information and Thermodynamics. Nature, 483,187-189 http://dx.doi.org/10.1038/nature10872

Bostock, D. (1988). Plato's Theaetetus. Oxford: The Clarendon Press.

Cifra, M., Fields, J., \& Farhadi, A. (2011). Electromagnetic Cellular Interactions. Progress in Biophysics and Molecular Biology, 105, 223-246.

Cucic, D. (2009). Paradoxes of Thermodynamics and Statiscal Physics. http://arxiv.org/ftp/arxiv/papers/0912/0912.1756.pdf 
De Grey, A. D. N. J., \& Rae, M. (2007). Ending Aging: The Rejuvenation Biotechnologies That Could Reverse Human Aging in Our Lifetime (416 p). New York, NY: St. Martin’s Press.

Hasan, T., Hernández-González, S., Dogbevia, G., Treviño, M., Bertocchi, I., Gruart, A., \& Delgado-Garcìa, J.-M. (2013). Role of Motor Cortex NMDA Receptors in Learning-Dependent Synaptic Plasticity of Behaving Mice. Nature Communications, 27.

Kak, S. (2013). Biological Memories and Agents as Quantum Collectives. Neuro Quantology, 11, 391-398. http://dx.doi.org/10.14704/nq.2013.11.3.682

Kirschvink, J., Kobashi-Kirschvink, A., \& Woodford, B. (1992). Magnetite Biomineralization in Human Brain. Proceedings of the National Academy of Sciences of the United States of America, 89, 7683-7687.

Loschmidt, J. (1876). Zur Grosse der Luftmoleciile, Sitzungsber. Kais.Akad.Wiss.Wien, Math. Naturwiss.Classe, 52, 395.

Pavlopoulos, E., Jones, S., Kosmidis, S., Close, M., Kim, C., Kovalerchik, O., Small, S., \& Kandel, E. (2013). Molecular Mechanism for Age-Related Memory Loss: The Histone-Binding Protein RbAp48. Science Translational Medicine, 5, 200ra115. http://dx.doi.org/10.1126/scitranslmed.3006373

Sirdeshmukh, D.B., Sirdeshmukh, L., \& Subhadra, K. G. (2001). Alkali Halides: A Handbook of Physical Properties (pp. 65, 68). Berlin: Springer. 